\section{IN THE NEWS}

One...two...many

According to The

Guardian (20 August

2004), "Researchers claim

to have solved the

mystery of the people

who simply do not count.

It could be because they

are lost for words." A

study of a small, isolated

hunter-gatherer tribe

from the Amazon has

shed light on the enduring

puzzle of whether people

can conceptualize

numbers without having

the language to describe them.

The Pirahã tribe has a counting system of only a few words: "one" (which can also mean "roughly one"), "two" and "many". Reporting in Science, Peter Gordon of Columbia University described that when asked to do a series of simple, non-verbal number-matching tasks with familiar objects (such as sticks, nuts and small batteries), "the ability of tribesmen to do [the tasks] faltered beyond two or three"

(The Telegraph, 20 August 2004).

Gordon claims that his work supports Whorf's hypothesis from the late 1930 s that language not only influences thought but can determine the way in which we think. Lisa Feigenson, a psychologist from Johns Hopkins University, hailed the study as "fantastic" and argued that "language must be causing the 'drastic' difference in the number sense of the Pirahã" (The Telegraph).

Brian Butterworth, a neuroscientist at University College London, finds the results surprising. Speaking to The Guardian, he said, "It has been known for 50 years that birds can match sets of up to about seven. So I find it very strange that these Pirahã adults are unable to do these tasks."

Alison Rowan

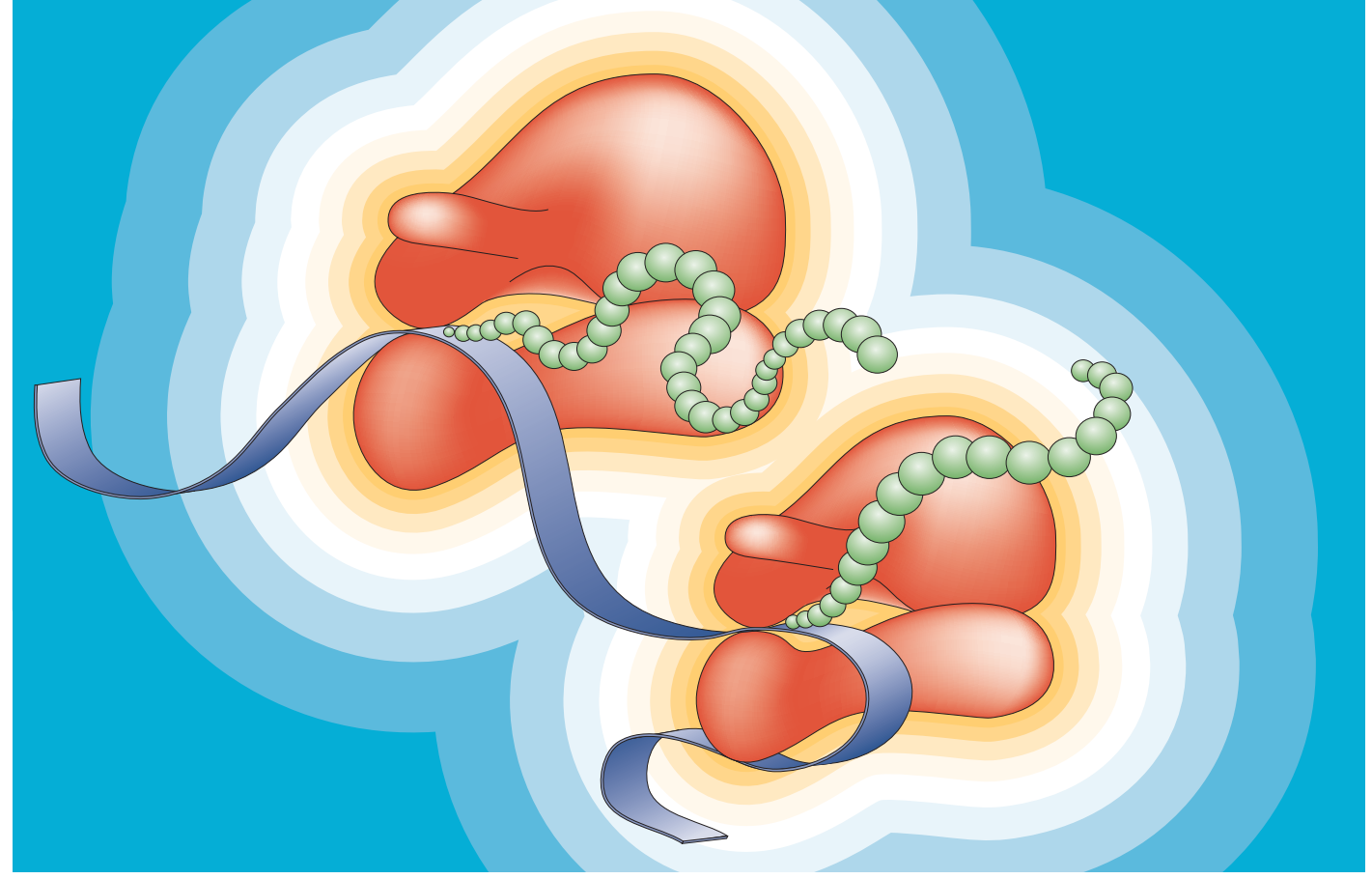

SYNAPTIC PLASTICITY

\title{
Found in translation
}

Over the past few years, it has become evident that local synthesis of proteins in axons and dendrites has a vital role in neural development and synaptic plasticity. We still know relatively little about how local protein synthesis is regulated, although there is some evidence that extracellular signals are involved. In a study reported in The Journal of Neuroscience, Schratt et al. present compelling new findings that indicate that brain-derived neurotrophic factor (BDNF) regulates the local translation of a defined subset of mRNAs at the synapse.

BDNF is known to stimulate the translation of several mRNAs in neurons, but it is unclear whether this represents a local or a global translational response. To investigate this phenomenon further, Schratt et al. carried out a genome-wide screen to identify mRNAs that became associated with the translational machinery in response to BDNF.

The authors isolated cortical neurons from the brains of embryonic day 18 rats, and cultured the neurons for 14 days to allow them to form synapses before exposing them to BDNF. They extracted mRNA from the cells, and centrifuged this preparation on a sucrose density gradient to separate out the fraction that was presumed to be undergoing active translation (the polysomal fraction). The authors then synthesized complementary DNA from this fraction, and hybridized it to an Affymetrix rat microarray. Out of about 3,500 mRNAs that were expressed by the neurons, as many as 143 entered the polysomal fraction in response to BDNF.

The authors further narrowed down their search to mRNAs that are regulated by BDNF at the level of transcription initiation. Specifically, they looked for cases in which BDNF-induced polysome association was prevented by rapamycin, an inhibitor of the mTOR-PI3K (mammalian target of rapamycinphosphatidylinositol 3-kinase) signalling pathway.
Forty-eight of the $143 \mathrm{mRNAs}$ fell into this category. To investigate whether BDNF was regulating local protein synthesis at the synapse, the authors focused on one of these mRNAs, Homer2, which encodes a postsynaptic adaptor protein. They found several lines of evidence to indicate that Homer2 translation was initiated in the dendritic compartment in response to BDNF. First, Homer2 mRNA was enriched in synaptoneurosomes (purified preparations of intact synapses), and the authors observed that its translation could be induced in synaptoneurosomes by the addition of BDNF. Second, in situ hybridization experiments showed that Homer2 mRNA was localized in the dendritic compartment of cultured hippocampal neurons. Last, sequences in the $3^{\prime}$ untranslated region of the Homer 2 mRNA were sufficient to target a green fluorescent protein-coding mRNA to the dendrites of hippocampal neurons in culture.

These findings strongly support the idea that BDNF stimulates the translation of a select group of mRNAs at the synapse, through a mechanism that includes the mTOR-PI3K pathway. The genomic approach that was developed for this study could be used to build up a profile of mRNAs that are translated in response to extracellular signals at various stages of development or synaptic plasticity. It should also provide new material with which to probe the mechanisms that enable certain mRNAs to be singled out for translation at specific sites.

Heather Wood

(2) References and links

ORIGINAL RESEARCH PAPER Schratt, G. M. et al. BDNF regulates the translation of a select group of mRNAs by a mammalian target of rapamycin-phosphatidylinositol 3-kinase-dependent pathway. J. Neurosci. 24, 7366-7377 (2004)

FURTHER READING Zhang, X. \& Poo, M. Localized synaptic potentiation by BDNF requires local protein synthesis in the developing axon. Neuron $\mathbf{3 6}$ 675-688 (2002) | Job, C. \& Eberwine, J. Localization and translation of mRNA in dendrites and axons. Nature Rev. Neurosci. 2, 889-898 (2001) 